Annuaire suisse de politique de développement

\title{
Partenariats public-privé et politique de développement
}

Bibliographie commentée

Judith Richter

\section{(2) OpenEdition}

\section{Journals}

Édition électronique

URL : http://journals.openedition.org/aspd/365

DOI : $10.4000 /$ aspd.365

ISSN : 1663-9669

\section{Éditeur}

Institut de hautes études internationales et du développement

Édition imprimée

Date de publication : 1 octobre 2005

Pagination : 193-213

ISSN : 1660-5934

\section{Référence électronique}

Judith Richter, "Partenariats public-privé et politique de développement », Annuaire suisse de politique de développement [En ligne], 24-2 | 2005, mis en ligne le 10 août 2010, consulté le 07 septembre 2020. URL : http://journals.openedition.org/aspd/365 ; DOI : https://doi.org/10.4000/aspd.365 


\title{
Partenariats public-privé et politique de développement
}

\section{Bibliographie commentée}

\author{
Judith Richter*
}

\begin{abstract}
$\mathrm{P}$
armi les débats actuels, rares sont ceux qui s'avèrent plus confus que celui sur le sens et l'utilité des partenariats public-privé (PPP, en anglais publicprivate partnerships) comme instrument de la politique de développement. L'une des raisons de cette confusion réside dans le fait que la notion de partenariat public-privé demeure extrêmement ambiguë. D'une part, les gouvernements, les organes de l'ONU et la Banque mondiale ne sont pas encore parvenus à s'entendre sur une définition commune. D'autre part, cette notion est très vague et imprécise. On l'utilise en effet non seulement pour se référer à plusieurs niveaux analytiques, mais aussi pour désigner les interactions les plus variées entre acteurs privés et publics.
\end{abstract}

L'expression «partenariat public-privé» désigne donc aussi bien un certain modèle de politique de développement que des initiatives et des interactions sur le plan pratique, de nature financière, décisionnelle ou opérationnelle. Elle englobe des activités traditionnelles telles que le sponsoring de projets et d'institutions par des entreprises et des fondations privées et les négociations de prix, mais aussi l'externalisation de services publics comme l'approvisionnement en eau et les services de santé. De plus, elle inclut également des alliances impliquant une participation du secteur privé, telles que l'Alliance mondiale pour les vaccins et la vaccination (Global Alliance for Vaccines and Immunization, GAVI), ainsi que les initiatives et les dialogues dits de parties prenantes multiples (multistakeholder initiatives and dialogues), visant par exemple à définir des codes volontaires de comportement pour les multinationales.

Malgré l'absence d'une définition claire et précise, la présente bibliographie s'est donné pour objectif de classer les documents existants selon leur contenu tout en les commentant avec un esprit critique. Ce classement devrait fournir aux lecteurs et aux lectrices du présent dossier de l'Annuaire suisse de politique de développement une vue d'ensemble des principaux documents qui traitent des partenariats public-privé dans la coopération internationale au développement.

Afin d'établir un classement critique, la présente bibliographie a été subdivisée en trois parties:

1. Origine et évolution des partenariats public-privé. Un nouvel instrument de la politique de développement;

2. Potentiels, risques et limites des partenariats avec le secteur privé, et alternatives au concept de partenariat;

* Chercheuse et auteure independante, Genève. 
3. Directives et mesures destinées à préserver les intérêts publics dans le cadre d'initiatives et d'interactions public-privé (public-private initiatives and interactions, PPI).

La première partie comprend surtout des documents et des déclarations politiques des Nations unies et des milieux proches de l'industrie, qui ont joué un rôle central dans l'introduction du nouveau modèle de coopération au niveau international. Elle distingue les documents de l'ONU, les documents issus de l'économie et les documents d'organisations hybrides.

La deuxième partie réunit des commentaires d'auteurs favorables au ou critiques envers le modèle de coopération que constituent les partenariats publicprivé. Elle comprend quelques documents techniques (de l'Union européenne, du Fonds monétaire international et de la Banque mondiale, en particulier sur les partenariats dans les secteurs des infrastructures et des services), ainsi que des publications qui considèrent ce modèle de coopération d'un point de vue plus large.

La troisième partie, enfin, tente de donner un aperçu des mesures dont s'est jusqu'ici dotée l'ONU et qui sont censées préserver les intérêts publics dans ses interactions avec les acteurs de l'économie privée. Y sont notamment présentées les Directives concernant la coopération entre l'Organisation des Nations unies et les entreprises, ainsi que deux publications qui devraient aider les collaborateurs de l'ONU à prendre des décisions d'ordre financier et autres lors d'interactions avec des acteurs de l'économie privée. Soulignons qu'il n'existe pas de site Internet qui permette d'accéder aisément à tous les documents de base que l'ONU a publiés sur ce sujet. Quelques-unes des directives et divers documents, pourtant fondamentaux, ne sont d'ailleurs pas accessibles au public. Cette situation ne devrait nullement empêcher les lecteurs et les lectrices de demander aux organes de l'ONU, au nom de la transparence et du mandat public confié à ces organismes, de mettre les différentes directives et le matériel pertinent à disposition.

Il est de toute évidence impossible, en une bibliographie commentée d'une vingtaine de pages, de passer en revue toute la littérature qui aborde les partenariats public-privé dans la politique de développement. A l'exception des documents consacrés au Pacte mondial de l'ONU, notre bibliographie ne comprend ainsi que quelques rares publications sur des initiatives concrètes qui figurent parmi les PPP. Les lecteurs et lectrices en quête d'autres documents sur le thème traité dans le présent Annuaire ne devraient dès lors pas se contenter de consulter les index thématiques sous «partenariats public-privé». Ils trouveront en effet aussi des ouvrages intéressants sous des mots clés tels que partenariat ONU-entreprises, initiatives de parties prenantes multiples, citoyenneté d'entreprise ou gouvernance multilatérale. Voici encore d'autres thèmes et mots clés: le problème des dons dans la politique de développement et ses projets; les partenariats de recherche et les conflits d'intérêts; la privatisation des services publics et de la politique; la responsabilité sociale des entreprises face à l'obligation de rendre compte (corporate social responsibility versus corporate accountability). On trouvera par ailleurs des informations sur la pertinence politique du partenariat comme paradigme de coopération sous des mots clés tels que restructuration/mondialisation néolibérale, rapport entre gouvernance mondiale en réseaux politiques et démocratie participative. Enfin, pour mieux comprendre le point de vue et les intérêts du secteur privé, il vaut la peine de lire des documents consacrés au marketing 
social, au sponsoring social stratégique, aux investissements philanthropiques selon le modèle californien, à la gestion de l'image et des risques.

Les documents présentés et les indications de la présente bibliographie commentée devraient encourager les scientifiques, les politiciens, les fonctionnaires de l'Etat et le public intéressé à sortir du cadre de pensée dominant pour s'interroger radicalement sur la nature, la nouveauté, l'utilité, le coût et les risques des partenariats avec l'économie privée, ainsi que sur des éventuelles alternatives. Il s'agit en fin de compte de remettre les intérêts des citoyens et des citoyennes au centre des préoccupations dans toutes les interactions entre institutions publiques et privées.

\section{Origine et évolution des partenariats public-privé. Un nouvel instrument de la politique de développement}

\section{Publications des Nations unies}

\section{Kofi Annan}

Le secrétaire général suggère aux dirigeants des grandes sociétés de conclure un «contrat mondial» avec les Nations unies pour donner un visage humain au marché mondial

Allocution prononcée le 31 janvier 1999 au Forum économique de Davos, Communiqué de presse SG/SM/6881, $1^{\text {er }}$ février 1999, <www.un.org/News/ fr-press/docs/1999/19990201.sgsm6881.html>, consulté le 10 février 2005.

Le discours du secrétaire général de l'ONU devant le Forum économique mondial à Davos illustre au mieux les circonstances dans lesquelles le Pacte mondial de l'ONU a vu le jour. Dans la plupart des milieux, ce discours a été compris comme un «défi» lancé par Kofi Annan aux dirigeants de l'économie afin de les inviter à conclure avec l'ONU «un "contrat mondial" fondé sur des valeurs et des principes communs qui donneront un visage humain au marché mondial». Voilà pourquoi ce pacte n'est souvent perçu que comme un contrat social prévoyant que l'économie intègre dans ses pratiques commerciales neuf principes figurant dans les droits de l'homme, les dispositions sur le droit du travail et les normes environnementales (complétés, depuis 2004, par un dixième principe relevant des normes anti-corruption).

Une lecture plus attentive du discours de Kofi Annan montre cependant que des contre-prestations ont été promises. Elles ont d'ailleurs revêtu une importance particulière aux yeux des représentants des multinationales, puisque ce discours a été prononcé à la veille des négociations de Seattle sur le commerce mondial. Parmi ces contre-parties, relevons avant tout la promesse faite par l'ONU de s'engager dans l'arène politique afin de contribuer à y faire régner «un environnement favorable au commerce et à l'ouverture des marchés ». Le secrétaire général a en outre promis que les organes de l'ONU aideraient les représentants de l'économie à engager un «dialogue» avec les groupements sociaux exigeant l'adoption de normes contraignantes sous forme d'accords sur le commerce et les investissements, afin de trouver d'autres solutions pour satisfaire les préoccupations de ces mouvements sociaux. 


\section{ONU}

\section{Coopération entre l'Organisation des Nations unies et tous les partenaires concernés, en particulier le secteur privé}

Point 50 de l'ordre du jour provisoire, «Vers des partenariats mondiaux», rapport du secrétaire général, doc. A/56/323, New York, 28 août 2001, 60 p.

Ce rapport de 2001 du secrétaire général (ainsi que le rapport qui a suivi en 2003, voir ci-après) a joué un rôle central dans les débats de l'Assemblée générale de l'ONU. Il a été établi pour donner suite à la résolution 55/215 de l'Assemblée générale, dans laquelle les Etats membres avaient mandaté le secrétaire général de s'enquérir de l'avis des Etats et des autres parties concernées sur les moyens et les possibilités d'engager une coopération plus étroite entre les organes de l'ONU et «tous les partenaires concernés, en particulier le secteur privé».

Comme bien d'autres documents, le rapport présenté en 2001 par le secrétaire général de l'ONU justifie la nécessité de mener une coopération plus étroite avec l'économie privée par l'augmentation du nombre d'acteurs non gouvernementaux, sans entrer dans les détails sur les différences fondamentales qui existent entre les groupes de la société civile, les entreprises axées sur le profit et encore d'autres acteurs du secteur privé (voir encadré 1 du rapport, «L'essor de la société civile et des entreprises privées », p. 4). Le rapport souligne qu'il convient de veiller à ce que la coopération avec des acteurs non étatiques n'altère en rien l'indépendance, la neutralité et l'intégrité de l'ONU, ni son caractère d'organisation dont les membres sont des Etats.

Le chapitre II résume le sondage d'opinion dont le secrétaire général avait été chargé. Il témoigne des questions, parfois très critiques, formulées par certains Etats membres et d'autres entités, par exemple sur la nature exacte et la définition de «partenariats» et sur la différence entre les partenariats et d'autres formes de coopération. Ce chapitre traite aussi d'une série de risques et de possibilités liés aux partenariats et répond à certains des problèmes soulevés (pp. 7-15).

Il est intéressant de relever que ce rapport ne donne encore aucune définition des «partenariats» avec l'économie et qu'il continue de parler de «coopération» ou de «relations» avec le secteur économique privé. Cette coopération est subdivisée en cinq catégories selon les fins poursuivies: le dialogue politique; les activités de plaidoyer; la mobilisation de fonds de source privée; l'information et l'apprentissage; et l'exécution au niveau des opérations ( $\$ 46$, p. 16). Le rapport contient cependant une définition du secteur privé, qui est largement détaillée dans son annexe ( 6 , p. 7; annexe I).

Le chapitre $\mathrm{v}$ décrit comment divers organes de l'ONU tentent d'intensifier leurs relations avec l'économie privée et comment ils font face aux risques que cela implique. Dans ce cadre, le chapitre renvoie aux Directives concernant la coopération entre l'Organisation des Nations unies et les entreprises, adoptées en juillet 2000 par le secrétaire général de l'ONU (annexe III; voir aussi la partie 3 de la présente bibliographie).

Un paragraphe de la conclusion mérite d'être repris tel quel: «La coopération entre l'Organisation des Nations unies et les acteurs non étatiques vise princi- 
palement à permettre à l'Organisation de servir plus efficacement les Etats membres et leurs peuples tout en se conformant aux principes de la Charte [de l'ONU]. Les activités de coopération devraient être périodiquement évaluées en fonction de ces objectifs. Partant, la coopération devrait être envisagée comme un moyen de parvenir aux buts de l'Organisation et d'améliorer son efficacité, et non comme une fin en soi » (§ 115, p. 36, italiques ajoutés).

Une partie de la confusion qui entache l'évaluation des projets de coopération et d'autres formes de relations avec les entreprises privées est à mettre sur le compte de ce rapport. En effet, c'est le Pacte mondial, et non pas la Charte de l'ONU, qui est présenté comme «un cadre général tout indiqué pour la coopération avec le milieu d'affaires», en particulier dans le choix des «partenaires commerciaux» (§ 118, p. 37; annexe III, §12, p. 54; italiques ajoutés).

\section{ONU}

Renforcement de la coopération entre l'Organisation des Nations unies et tous les partenaires concernés, en particulier le secteur privé

Point 47 de l'ordre du jour provisoire, «Vers des partenariats mondiaux», rapport du secrétaire général, doc. A/58/227, New York, 18 août 2003, 23 p.

Après avoir débattu en Assemblée générale du premier rapport mentionné cidessus, les Etats membres de l'ONU ont chargé le secrétaire général de présenter, dans un délai de deux ans, un autre rapport devant décrire plus en détail les conditions d'une coopération plus étroite avec le secteur privé.

Le rapport de 2003 se révèle particulièrement intéressant car il utilise cette fois le terme «partenariat» en lieu et place de «coopération». Il traite essentiellement des partenariats auxquels les organes de l'ONU participent soit en qualité de «partenaires», soit en tant que promoteurs. Le chapitre II, «Définition, concepts et modalités», en donne d'ailleurs une définition: «Par partenariat, on entend communément des relations de collaboration voulues [voluntary] entre diverses parties, Etats et autres acteurs, dans le cadre desquelles tous les participants acceptent de travailler ensemble à la réalisation d'un même but ou de s'engager dans une tâche bien précise en partageant les risques, les responsabilités, les ressources, les compétences et les avantages» (§9, p. 4).

Aux catégories définies dans le rapport précédent, celui de 2003 ajoute un autre type de coopération: la "promotion de partenariats».

Après avoir décrit l'évolution récente, le rapport se penche sur les «enseignements tirés de l'expérience» et les «enjeux à venir». Il n'aborde toutefois que de manière indirecte et dans un langage diplomatique les problèmes et les défis qui vont de pair avec une intensification de la coopération avec le secteur privé. Si le rapport ne fournit aucun fondement qui permettrait véritablement d'évaluer ce concept et les initiatives menées dans la pratique, une leçon en ressort néanmoins clairement: «Les partenariats doivent être justifiés du point de vue économique» (p. 17). Parmi les «enjeux à venir», le rapport souligne un nouveau rôle que les gouvernements et les Nations unies ont à jouer en tant que négociateurs et facilitateurs de partenariats, ainsi que comme promoteurs de la citoyenneté responsable des entreprises. 


\section{ICC, IOE}

Joint Written Statement Submitted by the International Chamber of Commerce and the International Organization of Employers, Non-Governmental Organizations in General Consultative Status

Commission on Human Rights, Sub-Commission on Promotion and Protection of Human Rights, Item 4, Economic, Social and Cultural Rights, doc. E/CN.4/Sub.2/2003/NGO/44, 29 juillet 2003, 3 p.

Une des questions clés du débat consacré aux «partenariats en politique» est de savoir s'il est possible de détourner le Pacte mondial pour miner les efforts visant à créer une législation internationale contraignante pour les entreprises transnationales. La Chambre de commerce internationale (CCI) et l'Organisation internationale des employeurs (OIE) ont répondu à cette question dans leur déclaration commune de juillet 2003, qui a été soumise à la Sous-Commission de la promotion et de la protection des droits de l'homme en parallèle au projet de Normes des Nations unies sur la responsabilité en matière de droits de l'homme des sociétés transnationales et autres entreprises.

La déclaration commune juge que les normes mentionnées ci-dessus entravent les efforts actuels de l'ONU pour encourager les entreprises à promouvoir et à respecter les droits de l'homme en participant au Pacte mondial ("counterproductive to the UN's ongoing efforts to encourage companies to support and observe human rights norms by participating in the Global Compact»). Selon cette déclaration, les normes risquent de susciter des réactions négatives de la part du milieu des affaires à un moment où les entreprises s'engagent toujours d'avantage de leur plein gré dans des initiatives devant inciter à un comportement responsable au sein du secteur privé (" [the draft norms] risk inviting a negative reaction from business, at a time when companies are increasingly engaging in voluntary initiatives to promote responsible business conduct»).

D'autres documents cruciaux émanant du secteur privé et ayant joué un rôle important dans l'élaboration et la définition des PPP sont entre autres cités dans les ouvrages - auxquels nous revenons plus loin - de Sandrine Tesner et Georg Kell (2001); Kenny Bruno et Joshua Karliner (2002); Ann Zammit (2003); et Judith Richter (2004).

\section{Organisations hybrides}

\section{Jane Nelson}

Business as Partners in Development: Creating Wealth for Countries, Companies and Communities

London, The Prince of Wales Business Leaders Forum in collaboration with The World Bank and The United Nations Development Programme (UNDP), 1996, 288 p.

Des recherches sur le rôle et l'influence d'organisations dites hybrides, telles que le Prince of Wales Business Leaders Forum (PWBLF) - rebaptisé The 
International Business Leaders Forum (IBLF) -, font largement défaut. L'ouvrage mentionné ici et les deux suivants visent à souligner l'influence que les entreprises peuvent exercer, au travers de ce type d'organismes proches de l'économie, sur la définition et l'encadrement des partenariats public-privé et sur l'élargissement de leur usage.

Selon le document, le Prince of Wales Business Leaders Forum a été fondé en 1990 par le prince de Galles en tant que réseau mondial regroupant les dirigeants économiques d'Europe, d'Amérique du Nord et du Sud, d'Afrique, du Moyen-Orient, d'Asie et du Pacifique. Le prince Charles est d'ailleurs aujourd'hui encore président de cette organisation officiellement enregistrée en Grande-Bretagne en tant qu'œuvre caritative. Au moment où l'ouvrage a été publié, le Conseil de surveillance de l'organisation réunissait des responsables d'entreprises telles qu'Asea Brown Boveri, BMW, BP, Coca-Cola et SmithKline Beecham. La majeure partie des ressources financières du PWBLF provient également d'entreprises privées.

Très tôt déjà, cet organisme s'est donné pour principal objectif de négocier des partenariats entre l'économie privée et d'autres acteurs. Jane Nelson, aujourd'hui directrice de la section Business Leadership and Strategy de l'organisation, a rédigé l'ouvrage en 1996. Celui-ci résulte d'une initiative commune du PWBLF, de la Banque mondiale et du Programme des Nations unies pour le développement (PNUD).

Le livre de Jane Nelson fait œuvre de précurseur et mérite d'être lu notamment parce qu'il énumère les avantages que les entreprises peuvent tirer des «partenariats conjoints». L'auteure mentionne par exemple l'amélioration de l'image et la liberté de mener certaines activités, un positionnement stratégique sur le marché et la promotion d'une meilleure gestion des risques (pp. 7 et 52-54). La partie consacrée aux investissements sociaux et à la philanthropie s'avère particulièrement intéressante. Elle explique en effet que nombre d'entreprises ont renoncé à la philanthropie réactive, pour lui préférer une évaluation proactive des effets et une gestion du partenariat mieux ciblée sur les objectifs économiques. Une partie de cette évolution viserait à développer une approche plus stratégique de la philanthropie et, dans certains cas, à renoncer tout simplement à ce terme pour le remplacer par des notions telles qu'«investissement communautaire » et «partenariat» (pp. 65-76).

\section{Jane Nelson}

\section{Building Partnerships: Cooperation between the United Nations System and the Private Sector}

Report commissioned by the United Nations Global Compact, New York, United Nations Department of Public Information, 2002, 352 p.

Quelques années plus tard, Jane Nelson a rédigé un livre sur les partenariats de coopération entre le système des Nations unies et l'économie privée. Commandé par le Bureau du Pacte mondial, cet ouvrage résulte d'une initiative commune du Pacte mondial, du Département de l'information des Nations unies et du Prince of Wales Business Leaders Forum. Sur ses sept chapitres, les cinq premiers se fondent sur le rapport Coopération entre l'Organisation des Nations unies et tous les partenaires concernés, en particulier 
le secteur privé présenté en novembre 2001 par le secrétaire général de l'ONU à l'Assemblée générale (voir ci-dessus).

Le travail de Jane Nelson a consisté à développer le rapport du secrétaire général, de manière à faire des informations réunies un guide pratique qui fournit un aperçu des différents types de relations entre les organes de l'ONU et l'économie privée. L'ouvrage présente d'ailleurs plus de 150 exemples de partenariats entre l'ONU et l'économie privée. L'auteure établit une typologie de dix catégories de partenariats (voir en particulier pp. 11 et 43-45).

Jane Nelson propose en outre une définition des partenariats et tente de les distinguer d'autres types de coopération (voir encadré 8, «Defining Partnership», pp. 46-47). Elle souligne surtout la nature volontaire («voluntary nature») des partenariats et le processus de décision commun («the shared process of decision-making», p. 47). Pour terminer, le chapitre VI essaie de replacer les partenariats public-privé dans le contexte de la Déclaration du millénaire pour le développement, tandis que le dernier chapitre propose un plan d'action ( Agenda for Action») qui s'adresse aux entreprises, aux associations commerciales et économiques, aux organes de l'ONU et aux gouvernements.

La lecture de cet ouvrage s'impose pour tous ceux qui entendent se plonger dans ce sujet. D'une part, ce document reste aujourd'hui encore la «bible» attestée de l'ONU sur les partenariats public-privé, tout en illustrant le pouvoir du Prince of Wales Business Leaders Forum dans les définitions de leur notion politique. D'autre part, l'annexe contient des informations relatives aux principaux documents et sources sur le sujet (telle une liste des principaux sites Internet et références).

\section{Ros Tennyson, Luke Wilde}

\section{The Guiding Hand: Brokering Partnerships for Sustainable Development}

London; Turin, The Prince of Wales Business Leaders Forum; United Nations Staff College, 2000, 114 p.

Ce guide est une autre publication officielle à laquelle le PWBLF a largement participé. Il s'agit en effet du manuel officiel utilisé pour la formation des fonctionnaires internationaux de l'ONU au Staff College de l'ONU à Turin. Ros Tennyson et Luke Wilde décrivent ici comment les collaborateurs et les collaboratrices de l'ONU peuvent assumer au mieux leur rôle de négociateurs de partenariats tripartites. 


\section{Potentiels, risques et limites des partenariats avec le secteur privé, et alternatives au concept de partenariat}

\section{Littérature spécialisée}

\section{Commission des Communautés européennes}

Livre vert sur les partenariats public-privé et le droit communautaire des marchés publics et des concessions

Bruxelles, Commission des Communautés européennes, doc. COM (2004) 327 final, 30 avril 2004, 23 p., <http://europa.eu.int/eur-lex/lex/LexUriServ/site/fr/ com/2004/com2004_0327fr01.doc>.

En publiant son Livre vert, en avril 2004, la Commission des communautés européennes se proposait de lancer un large débat sur l'application du droit communautaire aux partenariats public-privé. Ce document ne s'interroge donc pas sur l'opportunité d'externaliser ou non certains services publics. Il vise plutôt à identifier les lacunes du droit communautaire à combler afin d'assurer aux acteurs économiques un meilleur accès aux différentes formes de partenariats public-privé dans des conditions de clarté juridique.

Comme l'explique l'ouvrage, il n'existe pas de définition communautaire des partenariats public-privé. Le communiqué de presse qui présente le Livre vert, le 4 mai 2004, définit les PPP comme suit: «Les partenariats publicprivé (PPP) sont des formes de coopération entre les autorités publiques et le monde des entreprises visant à satisfaire des besoins d'intérêt général. » Aux yeux de l'Union européenne, ils donnent lieu à des montages juridiques et financiers complexes, associant des opérateurs privés aux autorités publiques pour la réalisation de projets d'infrastructure ou la fourniture de services d'utilité publique.

Ces nouvelles structures économiques se seraient développées au cours des dix années précédentes dans divers pays de l'Union européenne, notamment dans le domaine du transport, de la santé publique, de l'éducation, de la distribution d'eau, de la gestion des déchets et de la sécurité publique. Le Livre vert explique le recours croissant aux PPP non seulement par les contraintes budgétaires, mais aussi par «la volonté de bénéficier davantage du savoirfaire et des méthodes de fonctionnement du secteur privé dans le cadre de la vie publique», ainsi que par «l'évolution plus générale du rôle de l'Etat dans la sphère économique, passant d'un rôle d'opérateur direct à un rôle d'organisateur, de régulateur et de contrôleur».

Le Livre vert propose de distinguer deux formes de partenariats public-privé:

$\square$ «les PPP de type purement contractuel, dans lesquels le partenariat entre secteur public et secteur privé se fonde sur des liens exclusivement conventionnels »;

๑ «les PPP de type institutionnalisé, impliquant une coopération entre le secteur public et le secteur privé au sein d'une entité distincte».

Cet ouvrage d'un abord relativement difficile présente surtout l'intérêt d'illustrer les conditions et l'absence de clarté juridique dans lesquelles nombre de pays de l'Union européenne externalisaient leurs services publics. 


\section{IMF}

\section{Public-Private Partnerships}

Prepared by the Fiscal Affairs Department (in consultation with other departments, the World Bank, and the Inter-American Development Bank), approved by Teresa Ter-Minassian, Washington D.C., International Monetary Fund (IMF), 12 March 2004, 48 p., <www.servicesforall.org/html/Privatization/IMF_Public_ Private_Partnerships.pdf>

Le titre de ce document du Fonds monétaire international paraît tout englober. Cependant, cette publication avait pour objectif premier de répondre à un grand souci propre aux milieux financiers: le risque que des PPP soient conclus pour contourner le contrôle sur les dépenses publiques.

La majeure partie du document présente donc les caractéristiques que devraient posséder des normes appropriées, exhaustives et reconnues au niveau international destinées à régir le contrôle économique et les déclarations fiscales, afin de garantir la transparence au sujet des coûts effectifs et des risques financiers. Le document donne par ailleurs un aperçu des instruments existants et propose l'application de mesures transitoires jusqu'à ce que les lacunes constatées soient comblées.

Ce document s'adresse aux spécialistes de l'économie et de la fiscalité, mais comprend une série de remarques et d'observations qui présentent aussi un intérêt pour le grand public.

Le FMI souligne également que les partenariats public-privé n'ont, à ce stade, pas encore été clairement définis. A ses yeux, les PPP désignent des accords dans lesquels le secteur privé fournit des infrastructures et des prestations économiques et sociales dont la mise à disposition incombait jusqu'alors aux pouvoirs publics. Considérant l'essor des PPP en rapport avec la privatisation, le FMI pense que les PPP seraient apparus lorsque la vague de privatisations des années 1990 a perdu de son élan.

Le FMI souhaiterait limiter la notion de partenariats public-privé aux accords correspondant au schéma «design-build-finance-operate» (DBFO: concevoir-construire-financer-exploiter). Dans le cadre de ce schéma, les services étatiques déterminent quelles infrastructures ou prestations ils attendent du secteur privé. La différence par rapport au financement public traditionnel réside dans l'hypothèse selon laquelle le transfert de certains domaines de compétence - pour ce qui est de la conception, de la construction, du financement et de l'exploitation d'infrastructures - de l'Etat à des opérateurs privés augmente l'efficience.

Démontrant que les PPP n'améliorent pas automatiquement l'efficience, l'étude du Fonds monétaire international souligne aussi que les PPP n'ont jusqu'alors été soumis à aucune analyse économique exhaustive. Quant à affirmer que le secteur économique privé parvient à une efficience relativement supérieure, l'étude relève que les bases théoriques de cette affirmation s'avèrent contradictoires et que les recherches empiriques ont abouti à des résultats mitigés.

Les auteurs de l'étude tentent ensuite de décrire plus en détail les facteurs et les conditions institutionnelles dont il faut en particulier tenir compte pour 
déterminer si des PPP sont effectivement plus efficients et moins coûteux que des mesures étatiques traditionnelles. Ils se fondent en cela principalement sur des théories économiques néolibérales.

Relevons que pour illustrer la réussite de l'évaluation d'un PPP, l'étude cite en exemple la politique de l'Etat australien du Victoria. Or, en se penchant de plus près sur ce cas (encadré 2, p. 16), on constate que cet Etat ne présuppose nullement que l'application de l'économie de marché assure automatiquement une efficience plus élevée. Les directives édictées par cet Etat pour évaluer l'impact des PPP prennent en effet pour référence l'utilité publique (public benefits) et l'optimisation des ressources (value for money), et non pas l'efficience en tant que telle. Si l'Etat du Victoria opte pour un PPP, il incombe aux services gouvernementaux de veiller au respect des normes fixées et à la fourniture des prestations prévues. On mise ici avant tout sur la transparence.

En annexe, l'étude présente pour terminer les expériences faites par le Chili, l'Irlande, le Mexique et la Grande-Bretagne dans le domaine des partenariats public-privé. Ces études de pays se fondent essentiellement sur des informations fournies par les organes appropriés des pays en question. Et l'on ne peut pas attendre d'une étude du FMI qu'elle remette en cause l'évaluation positive de leurs propres programmes nationaux en matière de PPP. Les études comprennent toutefois de précieuses indications sur les points faibles de ces programmes ainsi que des informations qui permettent des interprétations divergentes. (Pour une étude de cas divergente des PPP en Grande-Bretagne, voir p. ex. l'ouvrage d'Allyson Pollock, ci-après.)

\section{Banque mondiale}

Rapport sur le développement dans le monde 2004. Des services pour les pauvres

New York, Oxford University Press, 2003, 210 p.

Rapport sur le développement dans le monde 2004. Des services pour les pauvres. Abrégé

Washington, D.C., Banque mondiale, 2003, 32 p., <www1.worldbank.org/ publications/pdfs/15638frontmat.pdf>

Les partenariats public-privé ne sont pas le sujet central du Rapport sur le développement dans le monde 2004, puisque celui-ci s'interroge sur les moyens d'améliorer l'accès aux services publics pour les groupes de population pauvres dans les pays en développement. Le rapport met surtout l'accent sur les soins de santé et l'éducation, qui sont considérés comme les deux piliers de la lutte contre la pauvreté.

Selon ses auteurs, l'édition 2004 du Rapport sur le développement dans le monde se fonde sur ses éditions précédentes. Ils mentionnent en particulier le rapport de 1993 sur la politique de santé publique, celui de 1994 sur l'amélioration des infrastructures et celui de 1997 sur le rôle de l'Etat dans la fourniture de certains services de base.

A y regarder de plus près, le rapport semble remettre en question nombre des solutions, dites techniques, préconisées par le passé. Il souligne notamment 
qu'un Etat serait malvenu de s'en remettre exclusivement au secteur privé pour fournir des prestations dans les domaines de la santé, de l'éducation, de l'approvisionnement en eau, de la gestion des déchets ou de l'approvisionnement en électricité. Le document remet ainsi à l'ordre du jour la question de savoir si - et, si oui, dans quelle mesure - les prestations doivent être fournies par le secteur privé ou, malgré tout, exclusivement assurées et contrôlées par le secteur public.

Le rapport recommande un modèle comprenant huit variantes, qui considère si le pays mène une politique au service des pauvres et tient compte d'autres facteurs tels que la difficulté de contrôler la qualité des services. Il propose de considérer les pauvres comme des clients et de leur donner les moyens d'exiger des services correspondant à leurs besoins.

Reste à savoir si ce modèle complexe à huit variantes constitue un progrès en matière de politique de développement. Le rapport de la Banque mondiale qualifie ce modèle de plus proche de la réalité et de plus souple que les modèles précédents; le problème réside toutefois dans le fait que l'analyse présentée dans le rapport mélange des exemples pris dans différents secteurs. Malgré ses efforts d'analyse contextuelle, la Banque mondiale retombe souvent dans des raisonnements purement économiques. De plus, elle omet d'autres analyses et discussions, pourtant d'importance, tel l'argument selon lequel les malades ne peuvent - et ne devraient - pas être considérés comme des «clients», car ils se trouvent dans une situation de dépendance asymétrique dont ils ne peuvent sortir tant qu'ils sont malades.

L'idéal consiste sans doute à considérer ce rapport comme une invitation à relancer le débat sur la manière la plus appropriée de garantir à tous les citoyens et à toutes les citoyennes un accès aux services de base.

\section{Littérature analytique}

\section{Tanja Brühl et al. (Hg.)}

Unternehmen in der Weltpolitik. Politiknetzwerke, Unternehmensregeln und die Zukunft des Multilateralismus

Texte der Stiftung Entwicklung und Frieden, Reihe Eine Welt, Bd. 19, Bonn, Verlag J.H.W. Dietz, 2004, 285 p.

Edité par le groupe de travail Global Policy de l'organisation allemande Weltwirtschaft, Ökologie und Entwicklung e.V. (WEED), cet ouvrage fait suite à celui publié en 2001 sous le titre Privatisierung der Weltpolitik (privatisation de la politique mondiale). Le premier volume avait conclu que l'engagement des ONG et des entreprises dans la politique mondiale sans réglementation allait priver le système de l'ONU de sa légitimité. L'une des questions demeurées en suspens portait sur la structure à donner au cadre politique afin d'obliger ces acteurs à contribuer à une politique mondiale démocratique et équitable.

Face à l'augmentation exponentielle du nombre de partenariats public-privé, le groupe de travail a décidé d'analyser ce problème en considérant tout d'abord l'économie privée. Ce volume réunit des articles originaux d'auteurs 
allemands critiques et des textes fondamentaux tirés du débat international. Dans la partie "Global Governance und Privatwirtschaft» (gouvernance mondiale et économie privée), Hartwig Hummel prévoit que si l'on ne poursuit pas l'effort visant à démocratiser et à légaliser l'engagement des réseaux privés et d'autres initiatives de partenariats, on assistera avant tout à l'apparition de «cartels élitaires». Après avoir analysé en détail la genèse du Pacte mondial, Ann Zammit met en évidence un paradoxe central de la notion de partenarial avec l'économie: Ces partenariats font «des principaux protagonistes du système néolibéral, qui [...] entrave le développement», les acteurs privilégiés appelés à résoudre les problèmes qui en résultent (voir aussi, d'Ann Zammit, Development at Risk, auquel nous revenons plus loin).

Dans la deuxième partie, Klaus Dingwert, Peter Utting et Andreas Wulf, notamment, se demandent s'il convient de considérer les réseaux politiques et les partenariats avec l'économie comme de futurs modèles du multilatéralisme.

Enfin, la partie intitulée «Jenseits der Freiwilligkeit: Ansätze für verbindliche internationale Unternehmenregeln» (au-delà des actions volontaires: vers des normes internationales contraignantes pour les entreprises) présente des approches existantes et prometteuses pour aborder le problème. David Weissbrod, l'un des principaux instigateurs des Normes des Nations unies sur la responsabilité en matière de droits de l'homme des sociétés transnationales et autres entreprises, évoque la naissance de ces normes, leur potentiel et leur avenir (encore incertain). D'autres contributions se penchent sur l'US Alien Tort Claims Act, la campagne Publish what you pay et les possibilités de contrer les pratiques fiscales dommageables des multinationales, cela afin de recouvrer des ressources pour accomplir les tâches publiques.

Ce volume a pour principal mérite de remettre en question les différentes interactions et initiatives classées jusqu'ici dans la catégorie des partenariats et de les replacer dans leur contexte idéologique et politique.

\section{Kenny Bruno, Joshua Karliner}

\section{Tangled up in Blue: Corporate Partnerships in the United Nations}

San Francisco, Transnational Resource \& Action Centre (TRAC), September 2000, $16 \mathrm{p}$.

Cette brochure de l'organisation américaine CorpWatch est sans doute une publication qui a beaucoup marqué les débats. Parue quelques mois à peine après le lancement officiel de la phase opérationnelle du Pacte mondial, elle présente de manière claire, succincte et intelligible la politique, l'idéologie et l'histoire des partenariats public-privé. Elle critique le Pacte mondial et les directives de l'ONU sur les PPP et propose des alternatives aux partenariats. Ce document reste surtout connu pour avoir créé le néologisme blue-washing («bleuissement»), qui souligne le risque d'un transfert d'image des organes de l'ONU (avec leurs logos et leurs drapeaux bleus) vers les partenaires privés. Les auteurs invitent le secrétaire général de l'ONU à envisager la création d'un «Pacte citoyen» (Citizens Compact) et de s'engager d'avantage en faveur de l'adoption d'une réglementation internationale contraignante sur les multinationales. 


\section{Kenny Bruno, Joshua Karliner}

\section{earthsummit.biz: The Corporate Take-over of Sustainable Development}

Oakland (California), Food First Books, 2002, 173 p.

Peu avant la conférence de Johannesburg, au cours de laquelle les PPP ont acquis une nouvelle légitimité sous le nom de outcome type II, Kenny Bruno et Joshua Karliner ont à nouveau tenté d'exercer une influence sur l'élaboration de la notion de partenariat comme paradigme de développement. Dans la première partie, intitulée «Perilous Partnerships» (partenariats à risques), ils documentent avec force explications et détails la continuité de la politique de partenariat avec le secteur privé menée par l'ONU depuis le Sommet mondial sur le développement durable (la CNUED, Conférence des Nations unies sur l'environnement et le développement) à Rio en 1992.

Les annexes comprennent des documents essentiels de l'Alliance for a Corporate-Free $U N$, alors très active, comme les lettres qu'elle a adressées au secrétaire général de l'ONU et le Pacte citoyen.

\section{Uwe Hoering}

«Public-Private Partnership: (nur ein) neues Instrument oder neue Epoche der Entwicklungspolitik?»

Peripherie, Jg. 18, Heft 72, 1998, pp. 6-24

\section{«Zauberformel PPP». «Entwicklungspartnerschaften» mit der Privatwirt- schaft. Ausmass - Risiken - Konsequenzen}

WEED Arbeitspapier, Bonn; Berlin, Weltwirtschaft, Ökologie und Entwicklung e.V. (World Economy, Ecology and Development [WEED]), octobre 2003, 46 p.

Les documents du journaliste Uwe Hoering possèdent une qualité particulière, celle de présenter la continuité dans l'évolution entre la politique de la Banque mondiale et l'introduction en Allemagne des partenariats publicprivé en tant qu'instrument prometteur de développement. Très tôt déjà, Uwe Hoering a soutenu que les PPP ne constituent pas simplement un nouvel instrument de développement, mais avant tout un produit de la réorientation néolibérale de la politique allemande de développement. Entamée par le ministre Carl Dieter Spranger, membre de la CSU, cette réorientation a d'ailleurs été poursuivie sans changement par le gouvernement de l'alliance rose-verte.

Se fondant sur des chiffres et des études de cas précis, Uwe Hoering démontre que nombre de PPP sont contraires aux valeurs et aux objectifs primordiaux de développement. Selon l'auteur, des PPP concrets se résumeraient souvent, lorsqu'on les considère de plus près, à une subvention des entreprises privées grâce aux fonds publics.

\section{Allyson Pollock}

\section{NHS plc: The Privatisation of Our Health Care}

London, Verso, 2004, $271 \mathrm{p}$.

Ce livre décrit les conséquences de l'introduction des partenariats publicprivé dans un secteur spécifique à l'échelle nationale. Avec ses collègues, 
Allyson Pollock, professeure de politique de la santé et chargée de recherche sur les services de santé au University College London, s'interroge sur les raisons et le processus qui ont conduit au morcellement du système de santé britannique, le célèbre National Health Service (NHS), puis à sa privatisation progressive. Les trois premiers chapitres de l'ouvrage décrivent comment le gouvernement conservateur, puis le gouvernement du New Labour, ont fait fi de tous les arguments, pourtant valables, s'opposant à leur projet et se sont attachés à privatiser un système de santé qui avait jusqu'alors servi de modèle à nombre de pays en développement.

Une part importante de cette privatisation progressive a commencé au début des années 1990 sous le nom de Private Finance Initiative (PFI), rebaptisée ensuite Public-Private Partnerships. Sur ce thème, il est particulièrement intéressant de lire comment, malgré les réglementations et le cadre définis par l'Etat, les PPP ont abouti à une restriction de l'accès de la population aux services de santé, tout en absorbant des fonds publics.

Le chapitre «Overcoming Opposition» mérite d'être lu, car il décrit les efforts entrepris pour dissimuler et réfuter les preuves allant à sens contraire. Cela explique pourquoi le débat tant national qu'international n'a pas encore considéré à leur juste valeur nombre des enseignements décrits ici.

\section{Social Watch}

\section{Social Watch Report 2003 : The Poor and the Market}

An international citizens' progress report on poverty eradication and gender equality, Montevideo (Uruguay), Instituto del Tercer Mundo, 2003, 199 p.

\section{Rapport de Contrôle citoyen 2003. Les pauvres et le marché}

Montevideo (Uruguay), Instituto del Tercer Mundo, $2003^{1}$.

Depuis 1996, le réseau Social Watch, un groupement d'organisations et d'institutions sociales et de politique de développement, suit la mise en œuvre des décisions prises à l'occasion du Sommet des Nations unies pour le développement social. Le Rapport de Contrôle citoyen 2003 a été rédigé pour faire pendant au Rapport sur le développement dans le monde 2004 publié par la Banque mondiale (voir ci-dessus).

Des articles thématiques et des rapports provenant de 52 pays du Sud et du Nord analysent les conséquences sociales et les processus politiques de la privatisation des institutions et des services publics. Dans un texte intitulé «Du contrat social aux contrats privés», Tim Kessler résume les principales conclusions des rapports par pays. La privatisation croissante des services publics de base (tels que l'eau, l'énergie et la santé) - souvent pratiquée sous le nom de partenariats public-privé - aurait conduit dans nombre de pays à une hausse des prix, à la dégradation de la qualité des services et à la marginalisation des pauvres. Les décisions politiques seraient par ailleurs souvent prises au terme de processus opaques et peu démocratiques. Tim Kessler

Pour commander l'édition en français du rapport, contacter ENDA Tiers-Monde, Programme SYSPRO2, BP 3370, Dakar, Sénégal, email <syspro2@enda.sn>. 
propose d'inscrire les services de base parmi les droits humains et de transférer la charge de la preuve des critiques de la privatisation des services publics à ceux qui préconisent qu'il faut «privatiser d'abord et poser des questions plus tard».

Le Rapport de Contrôle citoyen 2003 fournit des analyses solidement étayées des risques techniques et politiques que comporte la vague de privatisations. Ses arguments contre le modèle préconisé par la Banque mondiale méritent d'être lus et diffusés.

\section{Sandrine Tesner, with the collaboration of Georg Kell}

\section{The United Nations and Business : A Partnership Recovered}

New York, St. Martin's Press, 2000, 200 p.

Deux raisons rendent cet ouvrage incontournable: premièrement, il fournit un point de vue unique - interne - sur l'évolution des relations de partenariat de l'ONU et des débats ayant porté sur les lignes directrices entre 1997 et 2000. L'auteure, Sandrine Tesner, alors responsable des partenariats avec le secteur privé auprès du Bureau des Nations unies pour les services d'appui aux projets (UNOPS), disposait d'un accès unique tant aux documents et aux débats internes de l'ONU qu'aux personnalités les plus en vue du monde économique. C'est également le cas de Georg Kell, alors collaborateur au Cabinet du secrétaire général (et devenu depuis directeur exécutif du Pacte mondial). Il convient de louer ici l'attitude des auteurs, qui n'ont pas hésité, contrairement à ce que l'on constate dans nombre d'autres documents de l'ONU consacrés à ce sujet, à souligner et analyser aussi les événements et les aspects problématiques.

Deuxièmement, cet ouvrage constitue l'exemple par excellence d'un point de vue néolibéral en faveur d'un plus grand rapprochement entre le système des Nations unies et le secteur privé. La tentative de convaincre l'économie privée - mais aussi les Etats-Unis - des avantages du modèle de partenariat (et d'une attitude plus amicale à l'égard de l'ONU) se fonde en particulier sur la promesse de l'adoption éventuelle d'un modèle de politique internationale de développement qui correspondrait largement aux souhaits de l'économie privée: une organisation de l'activité économique qui favoriserait le rôle des entreprises, reposerait sur une responsabilité philanthropique et rejetterait toute intervention étatique ( optimal organization of economic activity - one that favors the role of business, relies on philanthropic responsibility, and shies away from government intervention », p. 154).

Les chapitres «The Road Ahead» et «Conclusion» présentent des idées sur la manière dont les partenariats pourraient représenter les intérêts de l'économie dans les forums des Nations unies (pp. 140-141 en particulier). Les auteurs soulignent l'importance politique du Pacte mondial en tant que précurseur de ce nouveau modèle de gouvernance multilatérale (p. 107). Selon eux, seule une structure tripartite de gouvernance (tripartite governance structure), qui réserverait une plus grande place à l'économie et aux organisations non gouvernementales, permettrait de garantir la survie du système de l'ONU. 


\section{Peter Utting \\ «Regulating Business via Multistakeholder Inititatives: A Preliminary Assessment"}

In R. Jenkins, P. Utting and R. Pino, Voluntary Approaches to Corporate Responsibility: Readings and a Resource Guide, NGLS Development Dossier, Geneva, UN Non-Governmental Liaison Service (NGLS); United Nations Research Institute for Social Development (UNRISD), 2002, pp. 61-126.

Cette contribution de Peter Utting, qui a dirigé pendant de longues années le projet international de recherche consacré à la responsabilité des entreprises dans le développement durable au sein de l'Institut de recherche des Nations unies pour le développement social (UNRISD), est un exemple représentatif des nombreux articles et documents publiés par cet institut.

Peter Utting se penche sur les raisons qui ont accru l'importance des initiatives de parties prenantes multiples pour la «coréglementation» (et la certification) de bonnes pratiques entrepreneuriales. Il analyse leurs points forts et leurs faiblesses en prenant quatorze exemples pratiques, tels que le Pacte mondial, la Global Reporting Initiative et le Marine Stewardship Council. Il parvient à la conclusion que de telles initiatives ne suffisent pas à elles seules pour garantir un comportement responsable et que les faiblesses et les limites de l' «autoréglementation» et de la «coréglementation» du secteur privé sont si évidentes que l'adoption de normes internationales sur les multinationales s'impose d'elle-même.

L'auteur relève que des réglementations nationales et des normes contraignantes n'ont souvent été adoptées par le passé que par réaction à une crise ou sous la pression de groupements de travailleurs ou d'autres mouvements sociaux. Pour que la communauté internationale complète les diverses initiatives dites «volontaires», il importe dès lors à ses yeux que les différents groupes sociaux réorientent leurs efforts pour exercer la pression nécessaire sur des organisations nationales et internationales compétentes.

\section{Ann Zammit}

\section{Development at Risk: Rethinking UN-business Partnerships}

Geneva, UNRISD in collaboration with South Centre, 2003, 328 p.

Aucun ouvrage n'a procédé à un examen aussi approfondi des partenariats entre l'ONU et le secteur privé que le livre d'Ann Zammit. En 328 pages, l'économiste et politologue décrit le contexte idéologique et historique qui a présidé à l'apparition et à l'essor des PPP pour en faire un nouveau paradigme de développement. Elle étudie leurs conséquences en prenant pour exemple les initiatives visant à promouvoir la responsabilité sociale des entreprises, tel le Pacte mondial, mais aussi des collaborations et des projets spécifiques, en particulier dans le domaine de la santé. Ann Zammit a le mérite de mettre le doigt sur la contradiction centrale que présentent les partenariats avec de grandes entreprises en tant que base du développement: "l'incohérence intrinsèque entre les intérêts politiques des pays en développement et les politiques préconisées par les partenaires économiques de l'ONU» («the basic inconsistency between the policy interests of developing countries and the policies promoted by the UN's corporate partners»). 
Elle décrit ainsi en détail les mesures qu'il faudrait appliquer pour mettre divers types de coopération et de relations avec l'industrie privée sur une voie qui tienne davantage compte des intérêts des pays en développement et qui respecte mieux leur souveraineté. L'auteure souhaite également que l'on soumette les partenariats actuels à une évaluation approfondie et que l'on dote les relations entre les Nations unies et l'économie privée d'un nouveau cadre politique. Dans le monde actuel, les entreprises ne sont toutefois plus à même d'assumer quelques-uns des nouveaux rôles que l'auteure leur attribue. On peut dès lors se demander s'il ne vaudrait pas mieux remplacer ces rôles «volontaires» par la promotion d'un cadre juridique contraignant et de la pression publique pour l'exiger.

Cet ouvrage devrait figurer dans la bibliothèque de toute personne un tant soit peu intéressée par la politique de développement.

\section{Directives et mesures destinées à préserver les intérêts publics dans le cadre d'initiatives et d'interactions public-privé (public-private initiatives and interactions, PPI)}

\section{ONU}

\section{Directives concernant la coopération entre l'Organisation des Nations unies et les entreprises}

Publiées par le secrétaire général de l'Organisation des Nations unies, New York, 17 juillet 2000, 7 p., <www.un.org/french/partners/business/otherpages/guide. htm>.

Ces directives du secrétaire général de l'ONU sur la coopération entre les Nations unies et les acteurs de l'économie privée ont été publiées en 2000, presque au même moment où l'ONU lançait la phase opérationnelle du Pacte mondial. Elles ont pour objectif de «faciliter la conception et la mise en œuvre de la coopération entre l'ONU et le monde des affaires, tout en préservant l'intégrité et l'indépendance de l'Organisation».

Les directives du secrétaire général doivent servir de cadre commun à toutes les institutions du système des Nations unies. Les organes de l'ONU sont par ailleurs encouragés à mettre au point des directives plus précises adaptées à leurs mandats et activités spécifiques. Selon les directives, la coopération avec les entreprises peut revêtir diverses formes, notamment «les activités de plaidoyer, la collecte de fonds, la concertation, l'assistance humanitaire et la coopération pour le développement», ainsi que de «nouvelles formes de partenariat» n'impliquant pas la participation active et directe du gouvernement.

Ces directives comprennent notamment des paragraphes définissant les principes généraux de la coopération, les critères présidant au choix des partenaires au sein de l'économie privée, des instructions relatives à l'utilisation du nom et de l'emblème de l'ONU, et d'autres modalités de coopération à respecter lorsque des organes de l'ONU concluent un partenariat avec des acteurs économiques privés.

La partie consacrée aux types d'arrangements recommande de distinguer les partenariats des activités d'approvisionnement, car celles-ci seraient régies 
par d'autres règles. Les partenariats sont ensuite subdivisés entre les contributions financières directes et indirectes de l'entreprise partenaire; les partenariats dans le cadre de projets d'assistance technique; les partenariats destinés à promouvoir les buts et activités de l'ONU; et les partenariats dans le cadre de projets de coopération.

La partie intitulée «Capacités institutionnelles» recommande aux organes de l'ONU de mettre en place les cadres et capacités institutionnelles nécessaires pour gérer ces arrangements de façon satisfaisante. Il s'agit par exemple de créer un centre de coordination chargé d'assurer la transparence de ces relations, de recueillir des informations et de promouvoir une meilleure compréhension du rôle et des objectifs des entreprises, en veillant à ce qu'ils soient compatibles avec les objectifs des Nations unies.

Les directives se terminent par un chapitre consacré aux «Examens futurs» ( «Future Reviews»), qui recommande de soumettre régulièrement les directives à un examen critique et de les mettre à jour pour conserver leur validité. Le texte ne définit pas l'intervalle de temps qui devrait séparer deux examens de ce type.

Depuis la publication de ces directives, toute une série d'organes et de fonds des Nations unies ont élaboré leurs propres directives et cadres de travail, mais les textes pertinents ne sont pas tous accessibles au grand public. $\mathrm{Ce}$ manque de transparence prive les Etats membres et les citoyens de la possibilité d'évaluer leur qualité en toute indépendance et de vérifier dans quelle mesure les différents organes de l'ONU respectent effectivement les directives qu'ils se sont données.

\section{Judith Richter}

Public-private Partnerships and International Health Policy Making: How Can Public Interests Be Safeguarded?

Elements for Discussion Series, Helsinki, Ministry for Foreign Affairs of Finland, Development Policy Information Unit, 2004, 105 p., <www.gaspp.org > ou $<$ www.globalfinland.fi $>$.

L'auteure de la présente bibliographie commentée a été chargée en 2003 de mener un projet de recherche afin d'évaluer la réussite des travaux d'élaboration de directives destinées à préserver les intérêts publics au sein de l'Organisation mondiale de la santé (OMS) - mais aussi d'autres forums se préoccupant de la santé publique, tels l'UNICEF et le Pacte mondial - et d'identifier les problèmes théoriques et pratiques rencontrés lors de la mise en place de procédures efficaces pour gérer les conflits d'intérêts dans les interactions public-privé.

Des entretiens avec des collaborateurs et des collaboratrices de l'ONU et l'analyse de nombreux documents ont montré que l'OMS a consacré dans ce domaine bien plus d'efforts que nombre d'autres organes de l'ONU. Le document explique le contexte dans lequel les différents instruments ont vu le jour et fournit les indications nécessaires pour permettre aux lecteurs et lectrices de consulter les directives en question. 
Malgré les efforts consentis, la réglementation de l'OMS destinée à préserver les intérêts publics dans le cadre d'interactions public-privé comporterait encore d'importantes lacunes. La directrice générale de l'OMS avait ainsi promis en 2001 qu'un cours de formation destiné aux fonctionnaires de l'OMS serait consacré aux conflits d'intérêts et à la gestion d'interactions ou d'initiatives public-privé. Or, à l'heure actuelle, même les collaborateurs de l'OMS ne peuvent plus se procurer la première étude consacrée à ce sujet. L'auteure du document a analysé un exemplaire de cette étude ainsi que les explications avancées pour son retrait de la circulation. Tout porte à croire que les raisons de ce retrait relèvent moins de la qualité de l'étude que de l'accent qu'elle met sur les conflits d'intérêts: on craignait en effet que cette préoccupation entrave la conclusion de partenariats plus «souples» dans le domaine de la santé.

La recherche menée a également identifié des lacunes dans les réglementations adoptées par l'initiative du Pacte mondial et le Fonds pour l'enfance (UNICEF), ainsi que dans leur application. Elle relève par ailleurs que certaines de ces lacunes ont eu des conséquences négatives dans le domaine de la santé.

Le document de réflexion propose des mesures que l'OMS et ses Etats membres pourraient appliquer pour mieux préserver les intérêts publics dans les initiatives public-privé. Il juge par ailleurs qu'il importe d'instaurer un débat franc et ouvert si l'on veut réexaminer et corriger l'ensemble de la réglementation.

A noter: quelques propositions de l'expert Eloy Anello en matière d'évaluation des conflits d'intérêts (assessing conflict of interest) figurent dans les publications suivantes: Yves Beigbeder, International Public Health: Patients' Rights vs. the Protection of Patents (Aldershot, Hants [England]; Burlington [Vermont], Ashgate, 2004), pp. 36-37, et Judith Richter, op. cit., pp. 24-31.

\section{OCDE}

\section{Gérer les conflits d'intérêts dans le service public. Lignes directrices de l'OCDE et expériences nationales}

Paris, Organisation de coopération et de développement économiques, 2005, $276 \mathrm{p}$.

L'ouvrage de l'Organisation de coopération et de développement économiques (OCDE) sur les moyens d'éviter et de gérer les conflits d'intérêts dans le service public pourrait s'avérer fort utile pour la révision des réglementations qui régissent les relations et la coopération entre les organes de l'ONU et les acteurs de l'économie privée. Il comprend notamment les Lignes directrices de l'OCDE pour la gestion des conflits d'intérêts dans le service public, qui ont été élaborées par des spécialistes sous la direction du Comité de la gestion publique de l'OCDE. Ces directives se fondent sur les principes et les politiques appliqués dans toute une série de pays membres de l'OCDE. Elles sont considérées comme «la première référence internationale destinée à faciliter aux gouvernements l'examen et l'élaboration de politiques globales relatives aux conflits d'intérêts dans le secteur public» (p. 23). 
Afin d'améliorer la compréhension générale des directives, l'ouvrage contient des comparaisons entre les législations pertinentes de différents pays de l'OCDE et quelques études nationales (par exemple pour le Canada, les Etats-Unis, la France et l'Allemagne).

L'OCDE recommande par ailleurs à ses trente Etats membres de revoir et de mettre à jour leur réglementation et leurs mesures nationales sur la base de ces directives. L'organisation souligne qu'il convient de réexaminer régulièrement les directives et les mesures destinées à gérer de manière satisfaisante les conflits d'intérêts dans le service public.

L'ouvrage souligne que les lignes directrices de l'OCDE se concentrent sur les conflits d'intérêts survenant au niveau des prises de décision individuelles. Il mentionne ainsi les lacunes suivantes: les conflits d'intérêts institutionnels, ainsi que les zones «grises» telles que les partenariats publicprivé, les programmes de privatisation ou de déréglementation, le parrainage par l'économie privée et les échanges de personnel entre secteurs (pp. 18, 22 et 31). Un des autres points faibles des directives de l'OCDE réside dans le fait qu'elles n'indiquent pas toujours clairement les valeurs fondamentales sur lesquelles doit se baser une appréciation des conflits d'intérêts.

Ces lacunes coïncident en partie avec celles des réglementations adoptées par diverses organisations internationales. L'OCDE a l'intention de les examiner de près d'ici mi-2006. Il serait utile que les représentants et représentantes des pays de l'OCDE qui prendront part à cette analyse gardent à l'esprit les situations qui n'ont pas encore été éclaircies dans le domaine international. 